\title{
Gap Functions and Algorithms for Variational Inequality Problems
}

\author{
Congjun Zhang, Baoqing Liu, and Jun Wei \\ College of Applied Mathematics, Nanjing University of Finance and Economics, Nanjing, Jiangsu 210023, China \\ Correspondence should be addressed to Baoqing Liu; lyberal@163.com
}

Received 7 July 2013; Accepted 10 October 2013

Academic Editor: Bo-Qing Dong

Copyright ( 2013 Congjun Zhang et al. This is an open access article distributed under the Creative Commons Attribution License, which permits unrestricted use, distribution, and reproduction in any medium, provided the original work is properly cited.

\begin{abstract}
We solve several kinds of variational inequality problems through gap functions, give algorithms for the corresponding problems, obtain global error bounds, and make the convergence analysis. By generalized gap functions and generalized D-gap functions, we give global bounds for the set-valued mixed variational inequality problems. And through gap function, we equivalently transform the generalized variational inequality problem into a constraint optimization problem, give the steepest descent method, and show the convergence of the method.
\end{abstract}

\section{Introduction}

Variational inequality problem (VIP) provides us with a simple, natural, unified, and general frame to study a wide class of equilibrium problems arising in transportation system analysis $[1,2]$, regional science [3, 4], elasticity [5], optimization [6], and economics [7]. Canonical VIP can be described as follows: find a point $x \in K \subset \mathbb{R}^{n}$ such that

$$
\langle T(x), y-x\rangle \geq 0, \quad \forall y \in K,
$$

where $K$ is a nonempty closed convex subset of $\mathbb{R}^{n}, T$ is a mapping from $\mathbb{R}^{n}$ into itself, and $\langle\cdot, \cdot\rangle$ denotes the inner product in $\mathbb{R}^{n}$.

In recent years, considerable interest has been shown in developing various, useful, and important extensions and generalizations of VIP, both for its own sake and for its applications, such as general variational inequality problem (GVIP) [8] and set-valued (mixed) variational inequality problem (SMVIP) [9]. There are significant developments of these problems related to multivalued operators, nonconvex optimization, iterative methods, and structural analysis. More recently, much attention has been given to reformulate the VIP as an optimization problem. And gap functions, which can constitute an equivalent optimization problem, turn out to be very useful in designing new globally convergent algorithms and in analyzing the rate of convergence of some iterative methods. Various gap functions for VIP have been suggested and proposed by many authors in $[8,10-13]$ and the references therein. Error bounds are functions which provide a measure of the distance between a solution set and an arbitrary point. Therefore, error bounds play an important role in the analysis of global or local convergence analysis of algorithms for solving VIP.

For the VIP defined in (1), the authors in [10] provided an equivalent optimization problem formulation through regularized gap function $G_{\alpha}: H \rightarrow \mathbb{R}$ defined by

$$
G_{\alpha}(x)=\max _{y \in K}\left\{\langle F(x), x-y\rangle-\frac{\alpha}{2}\|x-y\|^{2}\right\},
$$

where $\alpha$ is a parameter. The authors proved that $x$ is the solution of problem (1) if and only if $x$ is global minimizer of function $G_{\alpha}(x)$ in $K$ and $G_{\alpha}(x)=0$. In order to expand the definition of regularized gap function, the authors in [14] gave the definition of generalized regularized gap function defined by

$$
G_{\alpha}(x)=\max _{y \in K}\{\langle F(x), x-y\rangle-\alpha \phi(x, y)\},
$$

where $\phi$ is an abstract function which satisfies conditions ranked as follows:

(C1) $\phi$ is continuous differentiable on $H \times H$; 
(C2) $\phi$ is nonnegative on $H \times H$;

(C3) $\phi$ is uniformly strongly convex on $H$; that is, there exists a positive number $\lambda$ such that

$$
\begin{aligned}
\phi\left(x, y_{1}\right)-\phi\left(x, y_{2}\right) \geq & \left\langle\nabla_{2} \phi\left(x, y_{2}\right), y_{1}-y_{2}\right\rangle \\
& +\lambda\left\|y_{1}-y_{2}\right\|^{2}, \quad \forall x, y_{1}, y_{2} \in H
\end{aligned}
$$

(C4) $\phi(x, y)=0 \Leftrightarrow x=y$;

(C5) $\nabla_{2}(x, y)$ is uniformly Lipschtiz continuous on $H$; that is, there exists a constant $L^{\prime}>0$ such that

$\left\|\nabla_{2} \phi\left(x, y_{1}\right)-\nabla_{2} \phi\left(x, y_{2}\right)\right\| \leq L^{\prime}\left\|y_{1}-y_{2}\right\|, \quad \forall x, y_{1}, y_{2} \in H$.

Note that $\nabla_{2}$ is the partial of $\phi$ with respect to the second component and conditions (C1)-(C5) can make sense. One can refer to $[10,14]$ and so forth for more details.

Many gap functions have been explored during the past two decades as it is shown in [10-16] and the references therein. Motivated by their work, in this paper, we solve some classes of VIP through gap functions, give algorithms for the corresponding problems, obtain global error bounds, and make the convergence analysis. We consider generalized gap functions and generalized D-gap functions for SMVIP and give global bounds for the problem through the two functions, respectively. And for GVIP, we equivalently transform it into a constraint optimization problem through gap function, introduce the steepest descent method, and show the convergence of the method.

\section{Preliminaries}

Let $H$ be a real Hilbert space whose inner product and norm are denoted by $\langle\cdot, \cdot\rangle$ and $\|\cdot\|$, respectively. Let $K$ be a nonempty closed convex set in $H$ and let $2^{H}$ be the family of all nonempty compact subsets of $H$.

Let $F, f: H \rightarrow H$ be nonlinear operators. The GVIP can be described as follows: Find $x \in H, f(x) \in K$ such that

$$
\langle F(x), f(y)-f(x)\rangle \geq 0, \quad \forall y \in H, f(y) \in K .
$$

For single-valued operator $f: H \rightarrow \mathbb{R} \cup\{+\infty\}$, which is proper convex and lower semicontinuous, and for given multivalued operator $T: H \rightarrow 2^{H}$, the SMVIP can be described as follows: Find $x \in K, w \in T(x)$ such that

$$
\langle w, y-x\rangle+f(y)-f(x) \geq 0, \quad \forall y \in K .
$$

Note that when $f=0$, the original problem (7) reduces to a set-valued variational inequality problem; when $f=0$ and $T$ is a single-valued operator, problem (7) is the right problem discussed in (1).

Recall that the multivalued operator $T: K \subset H \rightarrow 2^{H}$ is said to be strongly monotone with modulus $\beta>0$ on $K$ if

$$
\begin{aligned}
& \left\langle w-w^{\prime}, x-x^{\prime}\right\rangle \geq \beta\left\|x-x^{\prime}\right\|^{2}, \\
& \forall(x, w),\left(x^{\prime}, w^{\prime}\right) \in \operatorname{graph}(T) .
\end{aligned}
$$

And $T$ is said to be Lipschtiz continuous on a nonempty bounded set $B \subset K$, if there exists a positive constant $L$ such that

$$
H(T(x), T(y)) \leq L\|x-y\|, \quad \forall x, y \in B,
$$

where $H(\cdot, \cdot)$ is the Hausdorff metric on $B$ defined by

$$
\begin{array}{r}
H(T(x), T(y)) \\
=\max \left\{\sup _{r \in T(x)} \inf _{s \in T(y)}\|r-s\|, \sup _{s \in T(y)} \inf _{r \in T(x)}\|r-s\|\right\}, \\
\forall x, y \in B .
\end{array}
$$

Let $F: \mathbb{R}^{n} \rightarrow \mathbb{R}^{n}$. Then $F$ is a $P_{0}$-function if $\max _{1 \leq i \leq n, x_{i} \neq y_{i}}\left(x_{i}-y_{i}\right)\left(F_{i}(x)-F_{i}(y)\right) \geq 0$, for all $x, y \in$ $\mathbb{R}^{n}$ and $x \neq y$. Assume $F_{\mu}(\cdot): \mathbb{R}^{n} \rightarrow \mathbb{R}^{n}(\mu>0) . F_{\mu}$ is called smoothing approximation function of $F$, if there exists a positive constant $k$ such that

$$
\left\|F_{\mu}(x)-F(x)\right\| \leq k u, \quad \forall u>0, x \in \mathbb{R}^{n} .
$$

And $F_{\mu}$ is a uniform approximation if $k$ is independent of $x$.

A matrix $M \in \mathbb{R}^{n \times n}$ is a $P_{0}$-matrix if each of its principal minors is nonnegative.

We need the following lemmas. The parameters involved in the lemmas can be found in the following sections.

Lemma 1 (see [11]). If abstract function $\phi$ satisfies condition (C1), then the following holds:

$$
\begin{array}{r}
\left\langle\nabla_{2} \phi\left(x, y_{1}\right)-\nabla_{2} \phi\left(x, y_{2}\right), y_{1}-y_{2}\right\rangle \geq 2 \lambda\left\|y_{1}-y_{2}\right\|^{2}, \\
\forall y_{1}, y_{2} \in H ;
\end{array}
$$

that is, $\nabla_{2}(x, \cdot)$ is strong monotone in $H$, and by (C5), one obtains that $2 \lambda \leq L^{\prime}$.

Lemma 2 (see [17]). If abstract function $\phi$ satisfies conditions (C1)-(C4), then

$$
\nabla_{2} \phi(x, y)=0 \Longleftrightarrow x=y .
$$

Lemma 3 (see [18]). If abstract function $\phi$ satisfies conditions (C1)-(C5) and $\lambda$ and $L^{\prime}$ are the corresponding coefficients defined above, then one has

$$
\lambda\|x-y\|^{2} \leq \phi(x, y) \leq\left(L^{\prime}-\lambda\right)\|x-y\|^{2}, \quad \forall x, y \in H .
$$

Lemma 4 (see [19]). If abstract function $\phi$ satisfies conditions (C1)-(C4), then $G_{\alpha}(x) \geq \alpha \lambda\left\|x-\pi_{\alpha}(x)\right\|^{2}$. Moreover, when $G_{\alpha}(x)=0, x$ is a solution of SMVIP.

Lemma 5 (see [10]). If abstract function $\phi$ satisfies conditions (C1)-(C4), then $g_{\alpha}(x)$ is differentiable and

$$
\begin{aligned}
\nabla g_{\alpha}(x)= & \nabla g(x) F(x)+\nabla F(x)\left(g(x)-y_{\alpha}(x)\right) \\
& -\alpha \nabla_{x} \phi\left(g(x), y_{\alpha}(x)\right) .
\end{aligned}
$$


Lemma 6 (see $[10,19])$. If abstract function $\phi$ satisfies conditions $(C 1)-(C 4)$, then $g_{\alpha}$ is nonnegative, and $g_{\alpha}(x)=0 \Leftrightarrow x$ is a solution of GVIP.

Lemma 7 (see [10]). Let abstract function $\phi$ satisfy conditions (C1)-(C4). If $\nabla g_{\alpha}(x)=0$ and $\nabla F(x)$ is positive definite, then $x$ is a solution of $\operatorname{GVIP}(F, f)$.

\section{Gap Functions and Error Bounds for SMVIP}

In this section, by introducing appropriate gap functions, we give global error bound for SMVIP. Firstly, we need the following propositions.

Proposition 8. Let $C$ be a nonempty closed convex set in $H$ and let $f$ be strictly convex in $C$. Then $f$ has only one minimum in $C$.

Proof. We use proof by contradiction to show the desired result. Let $x_{1}, x_{2} \in C$ be two minimal points of $f$; that is, $f\left(x_{1}\right)=f\left(x_{2}\right)=\min f(x)$. Since $f$ is strictly convex, one obtains that

$$
\begin{aligned}
f\left(\alpha x_{1}+(1-\alpha) x_{2}\right) & <\alpha f\left(x_{1}\right)+(1-\alpha) f\left(x_{2}\right) \\
& =f\left(x_{1}\right), \quad \forall \alpha \in(0,1) .
\end{aligned}
$$

This implies that there exists a point $x_{3}=\alpha x_{1}+(1-\alpha) x_{2} \epsilon$ $C$, such that $f\left(x_{3}\right)<f\left(x_{1}\right)$, which is a contradiction. This completes the proof.

Let $T, f$, and $\phi$ be defined as above and let $K$ be a nonempty closed convex set in $H$. Now, we can introduce generalized gap function $G_{\alpha}$ of $\operatorname{SMVIP}(T, K)$ defined as follows:

$$
\begin{array}{r}
G_{\alpha}(x)=\max _{y \in H} \Psi_{\alpha}(x, y) \\
=\max _{y \in H}\{\langle w, x-y\rangle+f(x)-f(y)-\alpha \phi(x, y)\}, \\
\forall x, y \in H, \alpha>0 .
\end{array}
$$

From uniform convex of $\phi(x, \cdot)$, one obtains that $-\Psi_{\alpha}(x, \cdot)$ is also uniform convex in $H$. By Proposition 8 , there exists a minimal point $\pi_{\alpha}(x)$ of $\phi(x, \cdot)$ in $H$, such that

$$
\begin{aligned}
G_{\alpha}(x)= & \left\langle w, x-\pi_{\alpha}(x)\right\rangle+f(x)-f\left(\pi_{\alpha}(x)\right) \\
& -\alpha \phi\left(x, \pi_{\alpha}(x)\right) .
\end{aligned}
$$

Proposition 9. If abstract function $\phi$ satisfies conditions (C1)(C4) and $f: H \rightarrow \mathbb{R} \cup\{+\infty\}$ is proper convex and lower semicontinuous, then for all $\alpha>0, x=\pi_{\alpha}(x) \Leftrightarrow x$ is a solution of $\operatorname{SMVIP}(T, K)$.

Proof. From the definition of $\pi_{\alpha}(x)$, one has

$$
\begin{aligned}
0 & \in \partial\left(-\Psi\left(x, \pi_{\alpha}(x)\right)\right) \\
& =w+\partial f\left(\pi_{\alpha}(x)\right)+\alpha \nabla_{2} \phi\left(x, \pi_{\alpha}(x)\right) .
\end{aligned}
$$

By the definition of subgradient, we have

$$
f(y) \geq f\left(\pi_{\alpha}(x)\right)-\left\langle w+\alpha \nabla_{2} \phi\left(x, \pi_{\alpha}\right), y-\pi_{\alpha}(x)\right\rangle,
$$

which is equivalent to

$$
\begin{aligned}
& \left\langle w, y-\pi_{\alpha}(x)\right\rangle+f(y)-f\left(\pi_{\alpha}(x)\right) \\
& \geq \alpha\left\langle-\nabla_{2} \phi\left(x, \pi_{\alpha}(x)\right), y-\pi_{\alpha}(x)\right\rangle .
\end{aligned}
$$

On the one hand, if $x=\pi_{\alpha}(x)$, from Lemma 2, one obtains $\nabla_{2} \phi\left(x, \pi_{\alpha}(x)\right)=0$, and so does $\alpha\left\langle-\nabla_{2} \phi\left(x, \pi_{\alpha}(x)\right), y-\right.$ $\left.\pi_{\alpha}(x)\right\rangle=0$. So, from (21), we have

$$
\left\langle w, y-\pi_{\alpha}(x)\right\rangle+f(y)-f\left(\pi_{\alpha}(x)\right) \geq 0,
$$

which implies that $x$ is a solution of $\operatorname{SMVIP}(T, K)$.

On the other hand, if $x$ is a solution of $\operatorname{SMVIP}(T, K)$, take $y=\pi_{\alpha}(x)$ in (7), then we have

$$
\left\langle w, \pi_{\alpha}(x)-x\right\rangle+f\left(\pi_{\alpha}(x)\right)-f(x) \geq 0 .
$$

From condition (C3), one has

$$
\begin{aligned}
& \phi(x, x)-\phi\left(x, \pi_{\alpha}(x)\right) \\
& \geq\left\langle\nabla_{2} \phi\left(x, \pi \pi_{\alpha}(x)\right), x-\pi_{\alpha}(x)\right\rangle \\
&+\lambda\left\|x-\pi_{\alpha}(x)\right\|^{2} .
\end{aligned}
$$

And by conditions (C2) and (C4),

$$
\phi(x, x)-\phi\left(x, \pi_{\alpha}(x)\right) \leq 0 .
$$

So we have

$$
\left\langle\nabla_{2} \phi\left(x, \pi_{\alpha}(x)\right), x-\pi_{\alpha}(x)\right\rangle+\lambda\left\|x-\pi_{\alpha}(x)\right\|^{2} \leq 0 .
$$

Combining (23) with (26), we have $x=\pi_{\alpha}(x)$. This completes the proof.

Based on the above discussion, one can obtain the following global error bound.

Theorem 10. If abstract function $\phi$ satisfies conditions (C1)(C5), $f$ is closed convex, and $T$ is strong monotone and Lipschtiz continuous with respect to the solution $\bar{x}$ of $\operatorname{SMVIP}(T, K)$, then one has

$$
\|x-\bar{x}\| \leq \frac{L+\alpha L^{\prime}}{\beta}\left\|x-\pi_{\alpha}(x)\right\|,
$$

where $L$ and $L^{\prime}$ can be found in (5) and (9), respectively.

Proof. Since $\bar{x}$ is a solution of $\operatorname{SMVIP}(T, K)$, take $\bar{w} \in T(\bar{x})$, then we obtain

$$
\langle\bar{w}, y-\bar{x}\rangle+f(y)-f(x) \geq 0 .
$$

Let $y=\pi_{\alpha}(x)$, for all $x \in H$. Then inequality (28) reduces to

$$
\left\langle\bar{w}, \pi_{\alpha}(x)-\bar{x}\right\rangle+f\left(\pi_{\alpha}(x)\right)-f(x) \geq 0 .
$$


Take $y=\bar{x}, \widehat{w} \in T(x)$ in (21) such that $\|\widehat{w}-\bar{w}\| \leq$ $H(T(x), T(\bar{x}))$. Then inequality (21) changes to

$$
\begin{array}{r}
\left\langle\widehat{w}, \bar{x}-\pi_{\alpha}(x)\right\rangle+f(\bar{x})-f\left(\pi_{\alpha}(x)\right) \\
\geq \alpha\left\langle-\nabla_{2} \phi\left(x, \pi_{\alpha}(x)\right), \bar{x}-\pi_{\alpha}(x)\right\rangle .
\end{array}
$$

Combining (29) and (30), we have

$$
\left\langle\widehat{w}-\bar{w}, \pi_{\alpha}(x)-\bar{x}\right\rangle \leq \alpha\left\langle\nabla_{2} \phi\left(x, \pi_{\alpha}(x)\right), \bar{x}-\pi_{\alpha}(x)\right\rangle .
$$

And note that

$$
\begin{aligned}
\alpha\left\langle\nabla_{2} \phi\left(x, \pi_{\alpha}(x)\right), \bar{x}-\pi_{\alpha}(x)\right\rangle & \alpha\left\langle\nabla_{2} \phi\left(x, \pi_{\alpha}(x)\right), \bar{x}-x\right\rangle \\
& +\alpha\left\langle\nabla_{2} \phi\left(x, \pi_{\alpha}(x)\right), x-\pi_{\alpha}(x)\right\rangle \\
= & \alpha\left\langle\nabla_{2} \phi\left(x, \pi_{\alpha}(x)\right)-\nabla_{2} \phi(x, x), \bar{x}-x\right\rangle \\
& -\alpha\left\langle\nabla_{2} \phi(x, x)-\nabla_{2} \phi\left(x, \pi_{\alpha}(x)\right), x-\pi_{\alpha}(x)\right\rangle \\
\leq & \alpha\left\|\nabla_{2} \phi\left(x, \pi_{\alpha}(x)\right)-\nabla_{2} \phi(x, x)\right\|\|\bar{x}-x\| \\
& -2 \alpha \lambda\left\|x-\pi_{\alpha}(x)\right\|^{2} \\
\leq & \alpha L^{\prime}\left\|x-\pi_{\alpha}(x)\right\|\|\bar{x}-x\| \\
& -2 \alpha \lambda\left\|x-\pi_{\alpha}(x)\right\|^{2} .
\end{aligned}
$$

From (8), one has

$$
\begin{aligned}
\beta \| x & -\bar{x} \|^{2} \\
\leq & \langle\widehat{w}-\bar{w}, x-\bar{x}\rangle \\
\leq & \left\langle\widehat{w}-\bar{w}, x-\pi_{\alpha}(x)\right\rangle+\left\langle\widehat{w}-\bar{w}, \pi_{\alpha}(x)-\bar{x}\right\rangle \\
\leq & L\|x-\bar{x}\|\left\|x-\pi_{\alpha}(x)\right\| \\
& +\alpha L^{\prime}\left\|x-\pi_{\alpha}(x)\right\|\|x-\bar{x}\| \\
\leq & \left(L+\alpha L^{\prime}\right)\|x-\bar{x}\|\left\|x-\pi_{\alpha}(x)\right\|,
\end{aligned}
$$

so we have

$$
\|x-\bar{x}\| \leq \frac{L+\alpha L^{\prime}}{\beta}\left\|x-\pi_{\alpha}(x)\right\| .
$$

This completes the proof.

Theorem 11. If abstract function $\phi$ satisfies conditions (C1)(C5) and T is strong monotone for the solution $\bar{x}$ of SMVIP and is Lipschtiz continuous with module $L$, then $\sqrt{G_{\alpha}}$ has global error bound with respect to SMVIP; that is,

$$
\|x-\bar{x}\| \leq \frac{L+L^{\prime}}{\beta \sqrt{\alpha \lambda}} \sqrt{G_{\alpha}(x)} .
$$

Proof. By Lemma 4 and Theorem 10, one obtains

$$
\begin{gathered}
G_{\alpha}(x) \geq \alpha \lambda\left\|x-\pi_{\alpha}(x)\right\|^{2}, \\
\|x-\bar{x}\| \leq \frac{L+\alpha L^{\prime}}{\beta}\left\|x-\pi_{\alpha}(x)\right\| .
\end{gathered}
$$

So we can obtain

$$
G_{\alpha}(x) \geq \frac{\alpha \lambda \beta^{2}}{\left(L+\alpha L^{\prime}\right)^{2}}\|x-\bar{x}\|^{2},
$$

which implies that

$$
\|x-\bar{x}\| \leq \frac{L+\alpha L^{\prime}}{\beta \sqrt{\alpha \lambda}} \sqrt{G_{\alpha}(x)} .
$$

This completes the proof.

Now, we introduce generalized D-gap function $H_{\alpha \gamma}$ for SMVIP which is defined by

$$
\begin{aligned}
H_{\alpha \gamma}(x)= & G_{\alpha}(x)-G_{\gamma}(x) \\
= & \max _{y \in H} \Psi_{\alpha}(x, y)-\max _{y \in H} \Psi_{\gamma}(x, y) \\
= & \left\langle w, \pi_{\gamma}(x)-\pi_{\alpha}(x)\right\rangle+f\left(\pi_{\gamma}(x)\right)-f\left(\pi_{\alpha}(x)\right) \\
& +\beta \phi\left(x, \pi_{\gamma}(x)\right)-\alpha \phi\left(x, \pi_{\alpha}(x)\right),
\end{aligned}
$$

where $\pi_{\alpha}(x)$ and $\pi_{\gamma}(x)$ are minimal points for $-\Psi_{\alpha}(x, \cdot)$ and $-\Psi_{\gamma}(x, \cdot)$ in $H$, respectively, and $0<\alpha<\gamma$. For $H_{\alpha \gamma}(x)$, we can conclude the following result.

Proposition 12. If abstract function $\phi$ satisfies condition (C3), then one has

$$
(\gamma-\alpha) \phi\left(x, \pi_{\gamma}(x)\right) \leq H_{\alpha \gamma}(x) \leq(\gamma-\alpha) \phi\left(x, \pi_{\alpha}(x)\right) .
$$

Proof. From the definition of $H_{\alpha \gamma}(x)$, one obtains that

$$
\begin{aligned}
H_{\alpha \gamma}(x)= & \max _{y \in H} \Psi_{\alpha}(x, y)-\max _{y \in H} \Psi_{\gamma}(x, y) \\
= & \Psi_{\alpha}\left(x, \pi_{\alpha}(x)\right)-\Psi_{\gamma}\left(x, \pi_{\gamma}(x)\right) \\
\geq & \Psi_{\alpha}\left(x, \pi_{\gamma}(x)\right)-\Psi_{\gamma}\left(x, \pi_{\gamma}(x)\right) \\
= & \left\langle w, x-\pi_{\gamma}(x)\right\rangle-\alpha \phi\left(x, \pi_{\gamma}(x)\right) \\
& -\left\langle w, x-\pi_{\gamma}(x)\right\rangle+\gamma \phi\left(x, \pi_{\gamma}(x)\right) \\
= & (\gamma-\alpha) \phi\left(x, \pi_{\gamma}(x)\right) .
\end{aligned}
$$

$H_{\alpha \gamma}(x) \leq(\gamma-\alpha) \phi\left(x, \pi_{\alpha}(x)\right)$ can be proved similarly. This completes the proof.

From Proposition 12, one has the following.

Proposition 13. If $\phi$ satisfies conditions (C1)-(C4), then $H_{\alpha \gamma}(x)$ is nonnegative, and $H_{\alpha \gamma}(x)=0 \Leftrightarrow x$ is a solution of $\operatorname{SMVIP}(T, K)$.

Proof. From Proposition 12 and nonnegative property of $\phi(\cdot, \cdot)$, we have that $H_{\alpha \gamma}(x)$ is nonnegative. 
On the one hand, if $H_{\alpha \gamma}(x)=0$, then by conditions (C2) and (C4), one has $x=\pi_{\alpha}(x)$. Then by Proposition 9, we conclude that $x$ is a solution of $\operatorname{SMVIP}(T, K)$.

On the other hand, if $x$ is a solution of $\operatorname{SMVIP}(T, K)$, by Proposition 9, one obtains that $x=\pi_{\alpha}(x)$. From condition (C4), one has $\phi\left(x, \pi_{\alpha}(x)\right)=0$. And since $H_{\alpha \gamma}(x)$ is nonnegative, we have $H_{\alpha \gamma}(x)=0$. This completes the proof.

By the generalized D-gap function, we have the following error bound for $\operatorname{SMVIP}(T, K)$.

Theorem 14. Let $\phi$ satisfy conditions (C1)-(C5). $T$ is strong monotone for the solution $\bar{x}$ of SMVIP and is Lipschtiz continuous with module $L$; then $H_{\alpha \gamma}(x)$ has global error bound with respect to SMVIP; that is,

$$
\|x-\bar{x}\| \leq \frac{L+L^{\prime}}{\beta \sqrt{\lambda(\gamma-\alpha)}} \sqrt{H_{\alpha \gamma}(x)} .
$$

Proof. From Lemma 3, Theorem 10, and Proposition 13, we have

$$
\begin{aligned}
H_{\alpha \gamma}(x) & \geq(\gamma-\alpha) \phi\left(x-\pi_{\gamma}(x)\right) \\
& \geq(\gamma-\alpha) \lambda\left\|x-\pi_{\gamma}(x)\right\|^{2} \\
& \geq \lambda(\gamma-\alpha)\left(\frac{\beta}{L+\alpha L^{\prime}}\right)^{2}\|x-\bar{x}\|^{2},
\end{aligned}
$$

which implies that

$$
\|x-\bar{x}\| \leq \frac{L+L^{\prime}}{\beta \sqrt{\lambda(\gamma-\alpha)}} \sqrt{H_{\alpha \gamma}(x)} .
$$

This completes the proof.

\section{Steepest Descent Method for GVIP}

In this section, by introducing appropriate generalized gap function, the original $\operatorname{GVIP}(F, f)$ in $(6)$ can be changed into an optimization problem with restrictions. When one designs algorithms to solve the optimization problem, the gradient of objective function is unavoidable. We try to design a new algorithm, constructing a class of descent direction, to solve the optimization problem. In the following, we set $H$ to be $\mathbb{R}^{n}$. And we introduce the following generalized gap function for $\operatorname{GVIP}(F, f)$ :

$$
\begin{aligned}
g_{\alpha}(x) & =\max _{g(y) \in K} \Psi_{\alpha}(x, y) \\
& =\max _{g(y) \in K}\{\langle F(x), f(x)-f(y)\rangle-\alpha \phi(f(x), f(y))\} \\
& =\left\{\left\langle F(x), f(x)-y_{\alpha}(x)\right\rangle-\alpha \phi\left(f(x), y_{\alpha}(x)\right)\right\},
\end{aligned}
$$

where $y_{\alpha}(x)$ is a minimal point for $-\Psi_{\alpha}(x, \cdot), \alpha$ is a positive parameter, and $\phi$ satisfies conditions (C1)-(C5) stated above. For $g_{\alpha}$, we have the following useful results [14]:
(A1) $g_{\alpha}(x)$ is nonnegative in $K$;

(A2) $g_{\alpha}(x)=0$ for some $x \in K \Leftrightarrow x$ is a solution of VIP;

(A3) $y_{\alpha}(x)$ is the only minimizer of $\Psi_{\alpha}(x, \cdot)$ in $K$.

And similar to the discussion in $[10,11]$, we also give the following two assumptions:

(a) $\nabla F(x)$ is positive definite for all $x \in K$;

(b) $\nabla_{x} \phi(x, y)=-\nabla_{y} \phi(x, y)$.

From Lemmas 5-7, we obtain that the original GVIP (6) is equivalent to the following optimization problem:

$$
\min _{\text {s.t. } x \in K} g_{\alpha}(x) \text {. }
$$

For problem (46), we give the following algorithm.

Algorithm 15.

Step 0. Choose an initial value $x^{0} \in K, \varepsilon, t \in(0,1)$, and put $k=0$.

Step 1. If $g_{\alpha}(x) \leq \varepsilon$, then we can end the circulation.

Step 2. Compute $y_{\alpha}\left(x^{k}\right)$, and let

$$
d^{k}=y_{\alpha}\left(x^{k}\right)-f\left(x^{k}\right) .
$$

Step 3. Let $m_{k}$ be the minimal nonnegative integer $m$, such that

$$
g_{\alpha}\left(x^{k}+t^{m} d^{k}\right) \leq g_{\alpha}\left(x^{k}\right)-t^{2 m}\left\|d^{k}\right\|^{2} .
$$

Step 4. Let $f\left(x^{k+1}\right)=f\left(x^{k}\right)+t^{m_{k}} d^{k}, k=k+1$; go to Step 1 .

Proposition 16. Let $\left\{x^{k}\right\}$ be a sequence generated by Algorithm 15. If $\left\{x^{k}\right\}$ are not the solutions of $\operatorname{GVIP}(F, f)$, then

$$
\nabla g_{\alpha}\left(x^{k}\right)^{T} d^{k}<0
$$

that is, $d^{k}$ is the descent direction of $g_{\alpha}$ at $x^{k}$, where $d^{k}$ is defined in (47).

Proof. To begin, we show that $f\left(x^{k}\right) \in K$, for all positive integer $k$. From Algorithm 15, one obtains that $f\left(x^{0}\right) \in K$. We prove this result by induction. Assume $f\left(x^{k}\right) \in K$; we only need to show that $f\left(x^{k+1}\right) \in K$. Since $x^{k}, y_{\alpha}\left(x^{k}\right) \in K$, $t_{k} \in(0,1)$, and $K$ is convex, we have

$$
\begin{aligned}
f\left(x^{k+1}\right) & =f\left(x^{k}\right)+t_{k} d^{k} \\
& =\left(1-t_{k}\right) f\left(x^{k}\right)+t_{k} y_{\alpha}\left(x^{k}\right) \in K .
\end{aligned}
$$

For simplicity, $y_{\alpha}\left(x^{k}\right), x^{k}$ are replaced by $y_{\alpha}, x$, respectively. From Lemma 5, one has

$$
\begin{aligned}
\nabla g_{\alpha}(x)^{T} d \\
=\left\{y_{\alpha} F(x)+\nabla F(x)\left(g(x)-y_{\alpha}\right)-\alpha \nabla_{x} \phi\left(g(x), y_{\alpha}\right)\right\}^{T} d \\
=\left(g(x)-y_{\alpha}\right)^{T} \nabla F(x)\left(y_{\alpha}-g(x)\right) \\
\quad+\left\{\nabla g(x) F(x)-\alpha \nabla_{x} \phi\left(g(x), y_{\alpha}\right)\right\}^{T}\left(y_{\alpha}-g(x)\right) .
\end{aligned}
$$


Since $\left(g(x)-y_{\alpha}\right)^{T} \nabla F(x)\left(y_{\alpha}-g(x)\right)<0$, we only need to show that $\left\{\nabla g(x) F(x)-\alpha \nabla_{x} \phi\left(g(x), y_{\alpha}\right)\right\}^{T}\left(y_{\alpha}-g(x)\right) \leq 0$. Since $y_{\alpha}$ is the unique minimizer of $-\Psi(x, \cdot)$ in $K$, we have

$$
\begin{aligned}
& \left\langle-\nabla_{y} \Psi\left(x, y_{\alpha}\right), u-y_{\alpha}\right\rangle \\
& =\left\langle\nabla g(x) F(x)+\alpha \nabla_{y} \phi\left(x, y_{\alpha}\right), u-y_{\alpha}\right\rangle \\
& \quad \geq 0, \quad \forall u \in K .
\end{aligned}
$$

Let $u=x \in K$ in (52). One has

$$
\left\{\nabla g(x) F(x)+\alpha \nabla_{y} \phi\left(x, y_{\alpha}\right)\right\}^{T}\left(y_{\alpha}-x\right) \leq 0 .
$$

From assumption (b), we have

$$
\left\{\nabla g(x) F(x)-\alpha \nabla_{x} \phi\left(x, y_{\alpha}\right)\right\}^{T}\left(y_{\alpha}-x\right) \leq 0 \text {. }
$$

This completes the proof.

Now, we are in a position to show the global convergence result for Algorithm 15.

Theorem 17. Let $\left\{x^{k}\right\}$ be a sequence generated by Algorithm 15, and let $x^{\star}$ be the cluster point of $\left\{x^{k}\right\}$. Then $x^{\star}$ is a solution of $\operatorname{GIP}(F, f)$.

Proof. Let $\left\{x^{k}\right\}_{K}$ be a subsequence which converges to $x^{\star}$. If $g_{\alpha}\left(x^{\star}\right)=0$, then from Lemma $6, x^{\star}$ is a solution of GVIP. If $g_{\alpha}\left(x^{\star}\right) \neq 0$, from the continuous property, one obtains that $\left\{y_{\alpha}\left(x^{k}\right)\right\}_{K} \rightarrow y_{\alpha}\left(x^{\star}\right)$ which implies that

$$
\left\{d^{k}\right\} \longrightarrow y_{\alpha}\left(x^{\star}\right)-f\left(x^{\star}\right) .
$$

Now, we begin to show that the cluster point $d^{x^{\star}}$ of $\left\{d^{k}\right\}_{K}$ is zero. We use proof by contradiction. Assume $d^{\star}=y_{\alpha}\left(x^{\star}\right)-$ $x^{\star} \neq 0$. On the one hand, from Proposition 16, one has that

$$
\nabla g_{\alpha}\left(x^{\star}\right)^{T}<0
$$

On the other hand, from Proposition 16, we obtain that $\left\{g_{\alpha}\left(x^{k}\right)\right\}$ is monotonically decreasing and bounded; that is, the sequence $\left\{g_{\alpha}\left(x^{k}\right)\right\}$ is convergent. From step 3 of Algorithm 15, one has

$$
0 \leq t^{2 m_{k}}\left\|d^{k}\right\|^{2} \leq g_{\alpha}\left(x^{k}\right)-g_{\alpha}\left(x^{k+1}\right) \longrightarrow 0, \quad \text { as } k \longrightarrow \infty \text {. }
$$

Hence, we have $\lim _{k \rightarrow \infty} t^{2 m_{k}}\left\|d^{k}\right\|^{2}=0$; that is,

$$
\lim _{k \rightarrow \infty} t^{2 m_{k}}=0 \quad\left(d^{\star} \neq 0\right)
$$

Without loss of generality, we assume $t_{k} \in(0,1)$, for all $k$. Then one cannot find the minimal nonnegative integer $m_{k}$; that is,

$$
g_{\alpha}\left(x^{k}+t^{m_{k}-1} d^{k}\right)>g_{\alpha}\left(x^{k}\right)-\left(t^{m_{k}}\right)^{2}\left\|d^{k}\right\|^{2}, \quad \forall k,
$$

Or, equally,

$$
\frac{g_{\alpha}\left(x^{k}+t^{m_{k}-1} d^{k}\right)-g_{\alpha}\left(x^{k}\right)}{t^{m_{k}}}>-t^{m_{k}}\left\|d^{k}\right\|^{2}, \quad \forall k .
$$

Let $k \rightarrow \infty$, from (58), and $g_{\alpha}$ be continuous and differentiable; we can obtain

$$
\nabla g_{\alpha}\left(x^{\star}\right)^{T} \geq 0 \text {. }
$$

Inequalities (56) and (61) are at odds. This completes the proof.

\section{Acknowledgments}

The authors would like to thank the referees for the helpful suggestions. This work is supported by the National Natural Science Foundation of China, Contact/Grant nos. 11071109 and 11371198, the Priority Academic Program Development of Jiangsu Higher Education Institutions, and the Foundation for Innovative Program of Jiangsu Province Contact/Grant no. CXZZ12_0383.

\section{References}

[1] M. Florian, "Nonlinear cost network models in transportation analysis," Mathematical Programming Study, no. 26, pp. 167-196, 1986.

[2] M. J. Smith, "The existence, uniqueness and stability of traffic equilibria," Transportation Research B, vol. 13, no. 4, pp. 295304, 1979.

[3] M. Florian and M. Los, "A new look at static spatial price equilibrium model," Regional Science and Urban Economy, vol. 12, pp. 374-389, 1982.

[4] A. B. Nagurney, "Competitive equilibrium problems, variational inequalities and regional science," Journal of Regional Science, vol. 27, pp. 503-517, 1987.

[5] W. Han and B. D. Reddy, "On the finite element method for mixed variational inequalities arising in elastoplasticity," SIAM Journal on Numerical Analysis, vol. 32, no. 6, pp. 1778-1807, 1995.

[6] J. S. Pang and D. Chan, "Iterative methods for variational and complementarity problems," Mathematical Programming, vol. 24, no. 3, pp. 284-313, 1982.

[7] G. Cohen, "Nash equilibria: gradient and decomposition algorithms," Large Scale Systems, vol. 12, no. 2, pp. 173-184, 1987.

[8] M. A. Noor, "General variational inequalities," Applied Mathematics Letters, vol. 1, no. 2, pp. 119-122, 1988.

[9] S. C. Fang and E. L. Peterson, "Generalized variational inequalities," Journal of Optimization Theory and Applications, vol. 38, no. 3, pp. 363-383, 1982.

[10] M. Fukushima, "Equivalent differentiable optimization problems and descent methods for asymmetric variational inequality problems," Mathematical Programming, vol. 53, no. 1, pp. 99110, 1992.

[11] B. Qu, C. Y. Wang, and J. Z. Zhang, "Convergence and error bound of a method for solving variational inequality problems via the generalized D-gap function," Journal of Optimization Theory and Applications, vol. 119, no. 3, pp. 535-552, 2003.

[12] M. V. Solodov and P. Tseng, "Some methods based on the Dgap function for solving monotone variational inequalities," Computational Optimization and Applications, vol. 17, no. 2-3, pp. $255-277,2000$. 
[13] M. V. Solodov, "Merit functions and error bounds for generalized variational inequalities," Journal of Mathematical Analysis and Applications, vol. 287, no. 2, pp. 405-414, 2003.

[14] J. H. Wu, M. Florian, and P. Marcotte, "A general descent framework for the monotone variational inequality problem," Mathematical Programming, vol. 61, no. 3, pp. 281-300, 1993.

[15] J. S. Chen, "On some NCP-functions based on the generalized Fischer-Burmeister function," Asia-Pacific Journal of Operational Research, vol. 24, no. 3, pp. 401-420, 2007.

[16] M. A. Noor, "Merit functions for general variational inequalities," Journal of Mathematical Analysis and Applications, vol. 316, no. 2, pp. 736-752, 2006.

[17] N. Yamashita, K. Taji, and M. Fukushima, "Unconstrained optimization reformulations of variational inequality problems," Journal of Optimization Theory and Applications, vol. 92, no. 3, pp. 439-456, 1997.

[18] L. R. Huang and K. F. Ng, "Equivalent optimization formulations and error bounds for variational inequality problems," Journal of Optimization Theory and Applications, vol. 125, no. 2, pp. 299-314, 2005.

[19] Y. H. Hu, Gap gunctions and weak sharpness of solutions for variational inequalities [Ph.D. thesis], Southest Normal University, 2010 (Chinese). 


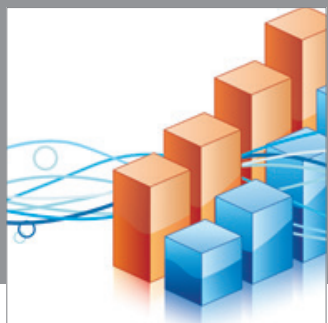

Advances in

Operations Research

mansans

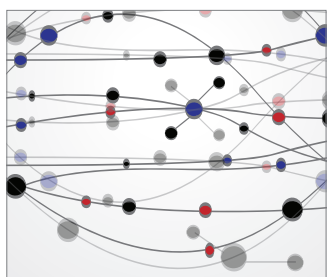

The Scientific World Journal
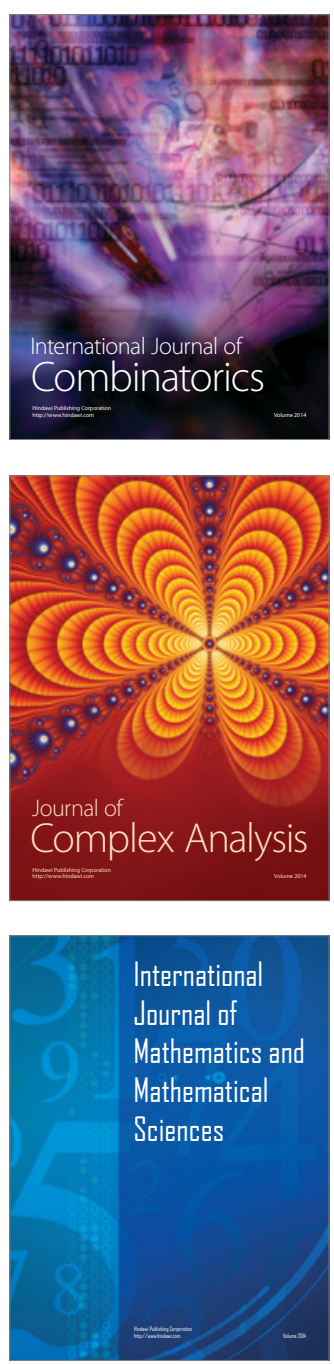
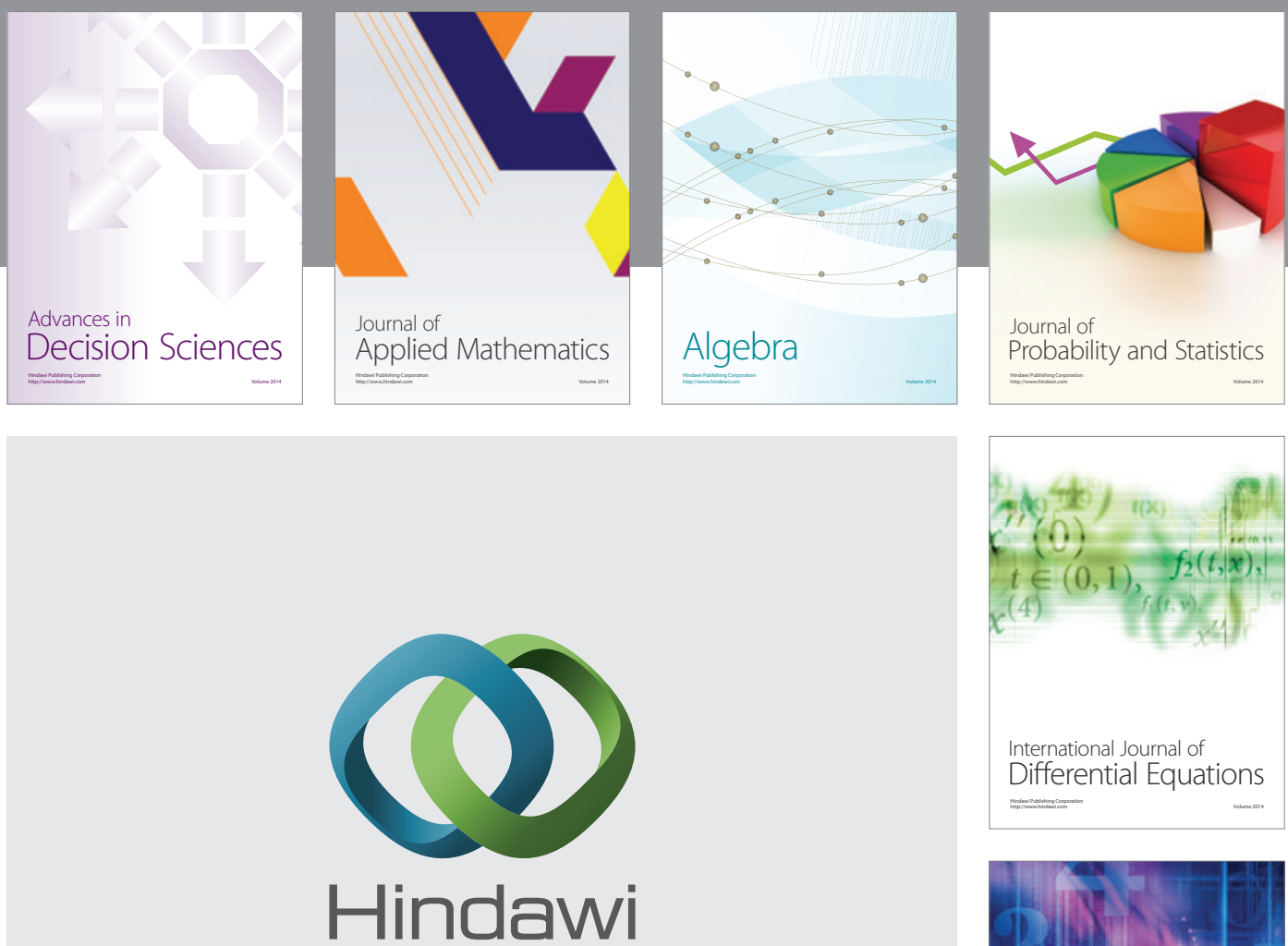

Submit your manuscripts at http://www.hindawi.com
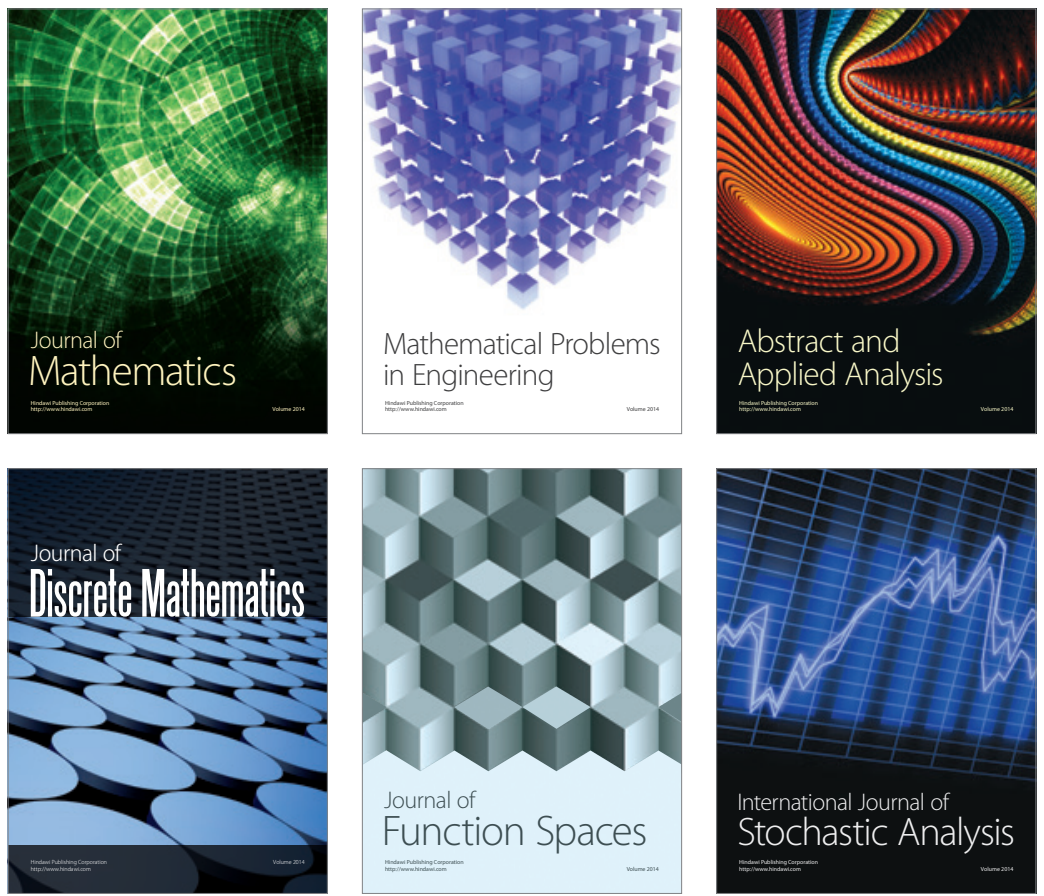

Journal of

Function Spaces

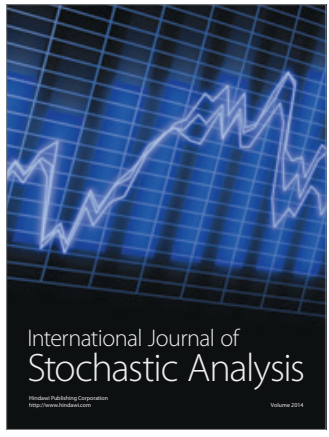

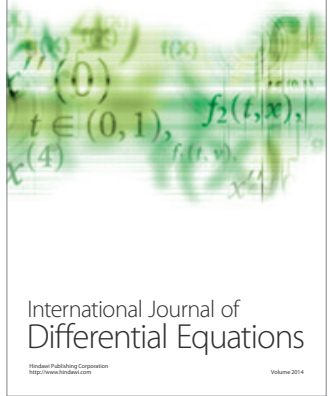
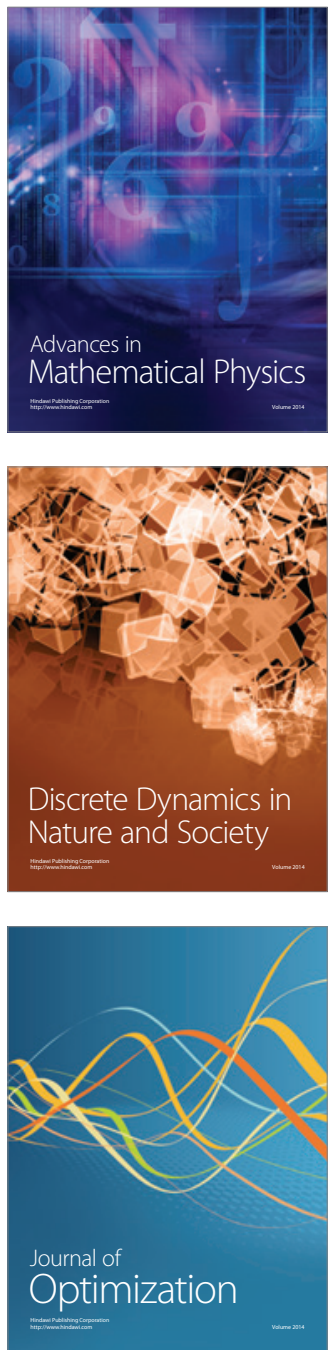